\title{
Research on Port-Starboard Discrimination Performance of Triplet Arrays under Different Noise Conditions
}

\author{
ZHANG Yu-ling ${ }^{1, a}$, ZHOU Sheng-zeng ${ }^{1, b}$, JIANG Xiao-yong ${ }^{1, c}$ \\ ${ }^{1}$ No.5200, JinDu Road, MinHang District, Shanghai, China \\ ayulingzh@163.com, bview222@sina.com, ${ }^{\mathrm{c}}$ jxy2007@live.cn
}

Keywords: triplet arrays, correlation coefficient, port-starboard discrimination, beamformer

\begin{abstract}
The triplet arrays provide the ability to solve the notorious port-starboard (PS) ambiguity problem of ordinary single line array. Cardioid beam pattern is provided by the triplet arrays structure by using the time delay of received signals and noise correlation model. The received noise is composed by ambient sea noise and self noise. The former is made of wind and shipping noise, the latter is made of towed flow noise and electric noise. The PS performance is worse when the flow noise increases. The correlation coefficient of triplet arrays is researched by simulation under different noise conditions. The minimum signal to noise ratio (SNR) of PS discrimination is analyzed for cardioid beamformer and adaptive beamformer. The ability to PS discrimination and anti-noise/jammer of adaptive beamformer is better than cardioid beamformer by processing simulation and trial data.
\end{abstract}

\section{Introduction}

The underwater target is detected, tracked, localized and classified through acoustic array and array signal process by sonar devices. The PS ambiguity problem can't be solved by ordinary single line array, because one hydrophone is omnidirectional. At the beginning of the 90 's, the triplet arrays are firstly introduced to solve PS ambiguity by Jean Bertheas. The method using noise correlation of triplet arrays is given by Y Doisy to solve PS ambiguity ${ }^{[1]}$. The three hydrophones of one triplet are treated as one directional hydrophones. Cardioid beampattern is provided by the triplet arrays structure by using the time delay of received signals and noise correlation model ${ }^{[2-5]}$ at the infinite PS rejection principle. Thus, the PS sides of the target can be discriminated. The PS discrimination performance of cardioid beamformer is optimum when the received noise is highly correlated. The PS discrimination and array gain is loss when the flow noise increases.

The adaptive beamformer of triplet arrays is a beam-domain beamformer. The weights are computed using the on-line estimated correlation matrix. If the correlation matrix is properly estimated, the ability of PS discrimination and array gain are optimum, and the sidelobe of PS beamformer is lower.

In the paper, the noise correlation coefficient under different noise conditions is researched by simulation, and meanwhile the PS discrimination performance is given for cardioid beamformer and adaptive beamformer.

\section{Principle of port-starboard discrimination of triplet arrays}

The layout-structured graphic of hydrophone triplet is shown in Fig.1, where $\theta$ is the angle between incoming signal and y axis, and $\phi$ is the angle between projection of incoming signal in xoz plane and $\mathrm{z}$ axis. Position of hydrophone triplet in the vertical plane is: $\left(\phi_{1}, \phi_{2}, \phi_{3}\right)=(0,-2 \pi / 3,2 \pi / 3)$. 


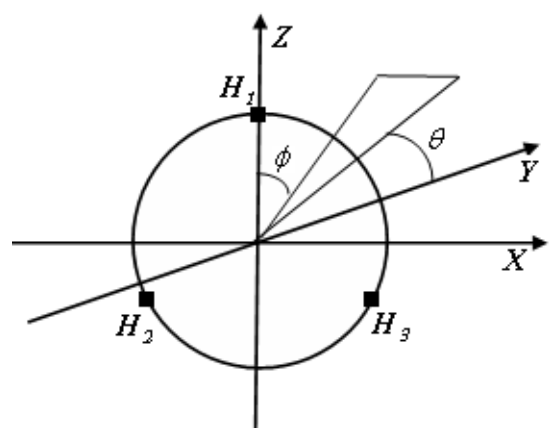

Fig.1 Inner structure of hydrophone triplet

The received signals, which contain signals from targets and complex noise, can be divided into two classes: non-correlation and correlation noise. The former is mainly flow noise and electrical noise, and the latter mainly includes ambient sea noise, shipping noise and traffic noise. The background noise of the triplet arrays is regarded as directive noise with the same correlation property. Thus, the noise correlation matrix of triplet arrays can be decomposed into two parts:

$$
\begin{aligned}
\mathbf{R}_{n} & =\sigma_{c}^{2} \mathbf{R}_{c}+\sigma_{u}^{2} \mathbf{I} \\
& =\sigma_{c}^{2}\left[\begin{array}{ccc}
1+\sigma^{2} & \alpha & \alpha \\
\alpha & 1+\sigma^{2} & \alpha \\
\alpha & \alpha & 1+\sigma^{2}
\end{array}\right]
\end{aligned}
$$

Where $\sigma^{2}=\sigma_{u}^{2} / \sigma_{c}^{2}$ is the variance ratio of non-correlation noise and ambient sea noise, and $\alpha$ is the correlation coefficient of ambient sea noise between adjacent hydrophones.

Based on signal detection theory, optimal beamformer coefficient of triplet arrays is derived from the generalized likelihood ratio criteria.

$$
\mathbf{w}=\frac{\mathbf{R}_{n}^{-1} \mathbf{e}}{\mathbf{e}^{H} \mathbf{R}_{n}^{-1} \mathbf{e}}
$$

Where $\boldsymbol{e}$ denotes the rotating vectors $\mathbf{e}_{+}=\mathbf{e}(\theta, \pi / 2)$ and $\mathbf{e}_{-}=\mathbf{e}(\theta,-\pi / 2)$, which are respectively port-starboard ambiguous direction.

The beamformer output of triplet arrays is:

$$
B(\theta, \phi)=\mathbf{w}^{H} \mathbf{b}(\theta)
$$

Where $\mathbf{b}(\theta)$ is a $3 \times 1$ dimension vector of conventional beamformer, and $\mathbf{w}$ is the weight vector of hydrophone triplet beamformer. Eq.2 is the beamformer coefficient of hydrophone triplets. The different methods are deduced at the different criteria, such as maximal array gain or maximal port-starboard rejection ratio.

\section{Simulation on correlation coefficient of triplet arrays under different noise conditions}

Correlation analysis of ambient sea noise and flow noise. Correlation is an effective method to separate the different noise effect based on different spatial correlation. Normally, ambient sea noise is regarded as spatial correlated, and correlation function is:

$$
\alpha(d)=\sin (k d) /\left.(k d)\right|_{d=r \sqrt{3}}, \quad k=2 \pi f / c
$$

The correlation function of flow noise is exponential function, and the function is:

$\beta(d)=\exp (-8 k d), \quad k=2 \pi f / c$

According to Eq.4 and Eq.5, $\alpha=0.99327$ and $\beta=0.2$ are obtained. The received noise is supposed as:

$$
S=\mathrm{A} 1 \times S_{\alpha}+\mathrm{A} 2 \times S_{\beta}
$$


Where A1 is the amplitude of ambient sea noise, and A2 is the amplitude of flow noise. $S_{\alpha}$ is ambient sea noise of triplet arrays, when the correlation coefficient $\alpha$ is equal to $0.99327 . S_{\beta}$ is flow noise of triplet arrays, when the correlation coefficient $\beta$ is equal to 0.2 .

$\eta$ is regarded as the energy ratio of flow noise and ambient sea noise, and when $\mathrm{A} 1=\mathrm{A} 2=1, \eta=$ $0 \mathrm{~dB}$. Supposing that $\mathrm{A} 1$ is equal to 1 , the correlation coefficient of triplet arrays $\rho$ is deduced at different $\eta$ with 3dB interval. The simulation results are shown in Table 1.

Table 1 Correlation coefficient of hydrophone triplets under different noise conditions

\begin{tabular}{|c|c|c|c|}
\hline$\eta$ & $\rho$ & $\eta$ & $\rho$ \\
\hline $0 \mathrm{~dB}$ & 0.5900 & $-3 \mathrm{~dB}$ & 0.7219 \\
\hline$+3 \mathrm{~dB}$ & 0.4600 & $-6 \mathrm{~dB}$ & 0.8375 \\
\hline$+6 \mathrm{~dB}$ & 0.3670 & $-9 \mathrm{~dB}$ & 0.9060 \\
\hline$+9 \mathrm{~dB}$ & 0.2840 & $-12 \mathrm{~dB}$ & 0.9464 \\
\hline$+12 \mathrm{~dB}$ & 0.2414 & $-15 \mathrm{~dB}$ & 0.9687 \\
\hline$+15 \mathrm{~dB}$ & 0.2318 & $-18 \mathrm{~dB}$ & 0.9809 \\
\hline$+18 \mathrm{~dB}$ & 0.2037 & $-21 \mathrm{~dB}$ & 0.9874 \\
\hline$+21 \mathrm{~dB}$ & 0.1993 & $-24 \mathrm{~dB}$ & 0.9900 \\
\hline
\end{tabular}

Correlation analysis in beam domain. Noise correlation coefficient from the object direction, which is closely related with SNR, is improved when the object is added in the received noise. In the section, a simulation example is presented by using triplet arrays data generated by $96 \times 3$ triplets. Assuming that correlation coefficient $\rho=0.3$, and the object is at the direction $60^{\circ}$, and SNR=-20dB. So correlation coefficient of triplet arrays in beam domain is shown as Fig.2. When an object with $\mathrm{SNR}=5 \mathrm{~dB}$ is added at the direction $15^{\circ}$, correlation coefficient from object direction is 0.73 , and correlation coefficient from supposed direction $15^{\circ}$ is 0.998 . Based on the simulation results, the effect of PS discrimination is not improved.

Supposing the object is at the direction $60^{\circ}$, and SNR varies from $-30 \mathrm{~dB}$ to $-10 \mathrm{~dB}$. When correlation coefficient $\rho$ is respectively $0.3,0.6,0.9$, the correlation coefficient at the direction $60^{\circ}$ is shown as Fig.3.

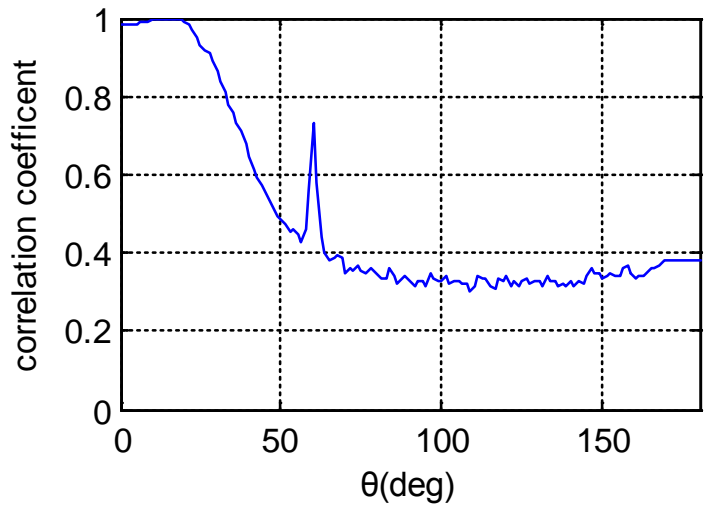

Fig.2 Correlation coefficient in beam domain

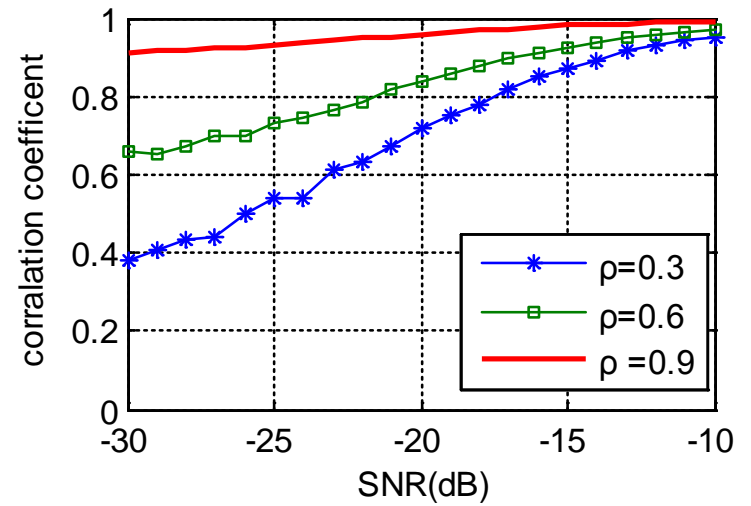

Fig.3 Correlation coefficient varying with SNR

\section{Adaptive triplet beamformer}

Basic principle. The sonar performance can be potentially improved by adaptive beamformer because of its high-resolution and anti-jammer ability.The triplet adaptive beamformer is a beam-domain beamformer. Its weights are deduced by using the actual estimated correlation matrix, while the weights of cardioid beamformer is obtained from using fixed coefficients. For every predefined beam direction, the weights are updated based on the correlation matrix of three line 
arrays. For one direction $\theta_{0}$, the beam outputs of triplet are defined $b_{1}\left(\theta_{0}\right), b_{2}\left(\theta_{0}\right), b_{3}\left(\theta_{0}\right)$, and the correlation matrix $\hat{\boldsymbol{R}}_{3 \times 3}$ can be estimated by Eq.7.

$$
\hat{\boldsymbol{R}}_{i j}\left(\theta_{0}\right)=\frac{b_{i}^{H} b_{j}}{\left|b_{i}\right| b_{j} \mid}
$$

Where $i, j \in[1,2,3]$ denote the number of three line array. After the correlation matrix is estimated, the adaptive beamformer can be proceeded using Eq.2 and Eq.3.

When the correlation matrix can be properly estimated under different noise conditions, the performance of cardioid beamformer is optimum. Firstly, the adaptive beamformer is equivalent to optimum beamformer with maximal array gain under the pure noise conditions. Secondly, at the direction of the target, the correlation increases by the target noise. The adaptive beamformer is trend to cardioid beamformer with the maximal PS rejection.

The flow of adaptive triplet beamformer. The flow of adaptive triplet beamformer is given by Fig.4. Firstly, the hydrophone element data is checked and calibrated. Secondly, the correlation matrix is estimated using the beam outputs of triplet arrays. After the adaptive beamfomer is performed using Eq.2, Eq.3, the panoramic azimuth beamformer output is given in the end.

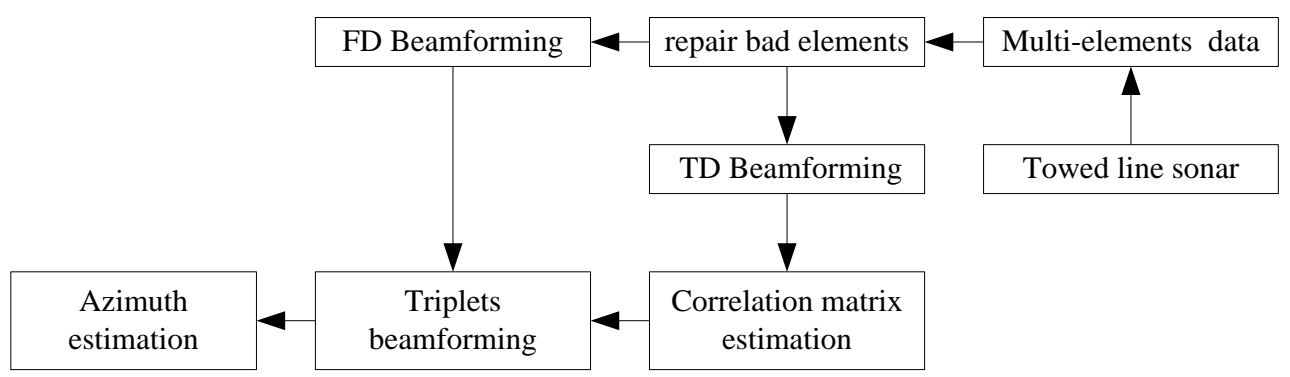

Fig.4 The flow of adaptive triplet beamformer

Simulation. In the section, we present an example on simulated data of triplet arrays generated by $96 \times 3$ triplets, and the correlation coefficient is 0.6 . The cardioid beamformer and adaptive triplet beamformer are tested on the synthetic data. Fig. 5 has shown the port-starboard discrimination performance with SNR varying from $-30 \mathrm{~dB}$ to $-15 \mathrm{~dB}$.

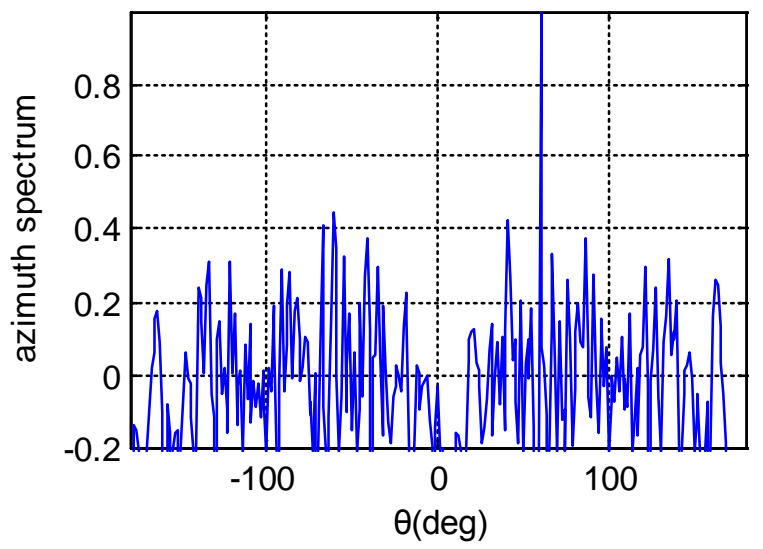

a) cardiod beamformer, $\mathrm{SNR}=-22 \mathrm{~dB}$

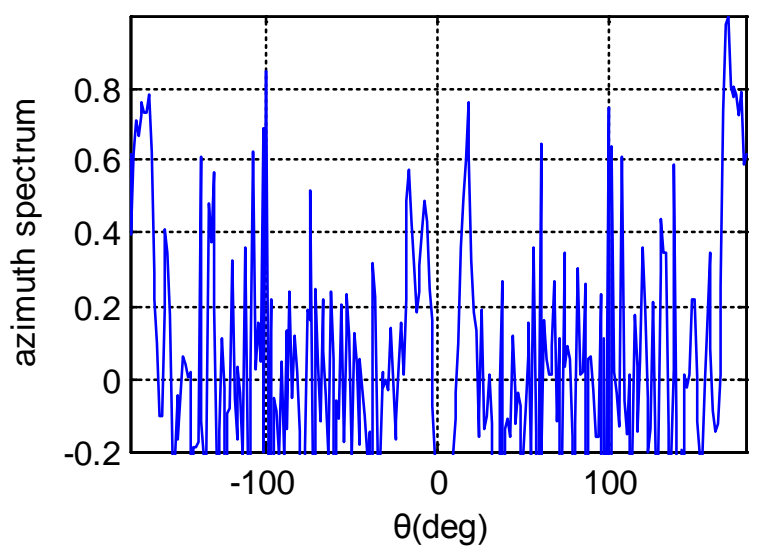

b) cardiod beamformer, $\mathrm{SNR}=-23 \mathrm{~dB}$ 


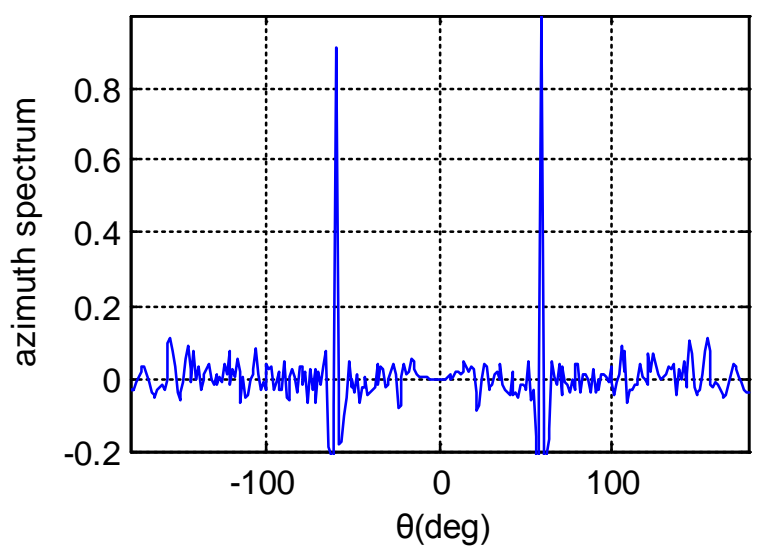

c) adaptive beamformer, $\mathrm{SNR}=-22 \mathrm{~dB}$

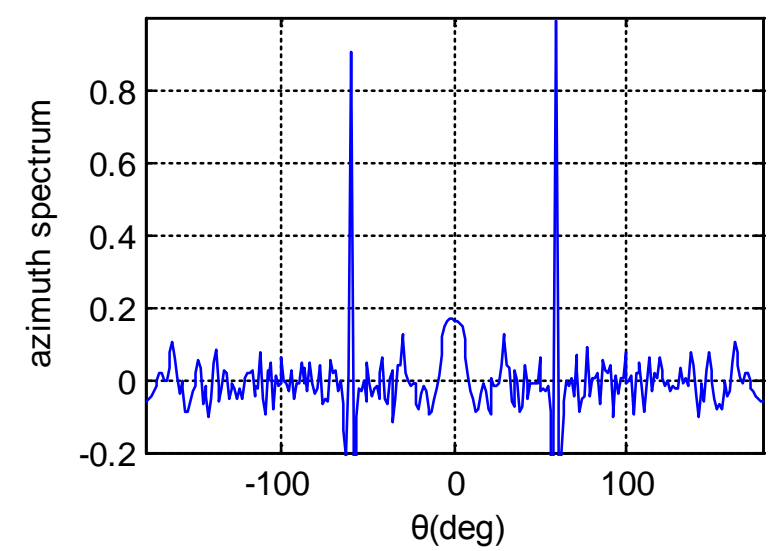

d) adaptive beamformer, $\mathrm{SNR}=-23 \mathrm{~dB}$

Fig.5 Azimuth estimation of the carioid and adaptive beamformer with different SNR.

In the upper panel of Fig.5, the cardioid beamformer is used. The object can be discriminated with $\mathrm{SNR}=-22 \mathrm{~dB}$. However, the object can not be discriminated with $\mathrm{SNR}=-23 \mathrm{~dB}$. In the lower panel of Fig.5, the adaptive beamformer is used. The object can be discriminated with both $\mathrm{SNR}=-22 \mathrm{~dB}$ and $\mathrm{SNR}=-23 \mathrm{~dB}$. Therefore, the port-starboard discrimination performance of the adaptive beamformer is optimum to the cardioid beamformer. The cardioid beamformer is optimum theoretically. But in practice, while the flow noise is dominant or the steering object is near the endfire, the adaptive beamformer has an advantage of the lower sidelobe and the higher ability to reject noises and jammers, except for much better port-starboard discrimination performance.

The actual noises received by triplet arrays consist of ambient sea noise and flow noise. The different proportion between ambient sea noise and flow noise affects the correlation coefficient. When the flow noise increases, the correlation coefficient is low. When the flow noise decreases, the correlation coefficient is high. Thus, the correlation coefficient of triplet arrays is an important factor to affect the port-starboard discrimination. In the simulation, we present an example on simulated data of triplet arrays generated by $96 \times 3$ triplets. The cardioid beamformer and the adaptive beamformer are tested on the synthetic data with the correlation coefficient varying from 0.2 to 0.9 . Table.2 has shown the minimum SNR of the port-starboard discrimination.

Tab.2 the port-starboard discrimination performance with different correlation coefficient

\begin{tabular}{|c|c|c|}
\hline $\begin{array}{c}\text { correlation } \\
\text { coefficient }\end{array}$ & $\begin{array}{c}\text { the minimum SNR of } \\
\text { cardioid beamformer(dB) }\end{array}$ & $\begin{array}{c}\text { the minimum SNR of } \\
\text { adaptive beamformer }(\mathrm{dB})\end{array}$ \\
\hline 0.2 & -18 & -26 \\
\hline 0.3 & -19 & -27 \\
\hline 0.4 & -20 & -27 \\
\hline 0.5 & -21 & -28 \\
\hline 0.6 & -22 & -28 \\
\hline 0.7 & -23 & -30 \\
\hline 0.8 & -25 & -31 \\
\hline 0.9 & -27 & -32 \\
\hline
\end{tabular}

Experiment Results. The sea-trial data are obtained from the triplet arrays. In the experiment, the cooperative object is at the direction $-67.5^{\circ}$. The correlation coefficient between the triplets is lower to 0.6, when the flow noise is very high during the towed course. Cardioid beamformer and adaptive triplet beamformer are used to analyze the sea-trial data. In Fig.6, the peak value from the adaptive triplet beamformer is detected at the direction $67.5^{\circ}$ on the port. However, the cooperative object can not be found in cardioid beamformer. Because the correlation coefficient between the triplets is very low, and the cardioid beamformer is based on noise correlation model, so it can not discriminate the port- starboard. 


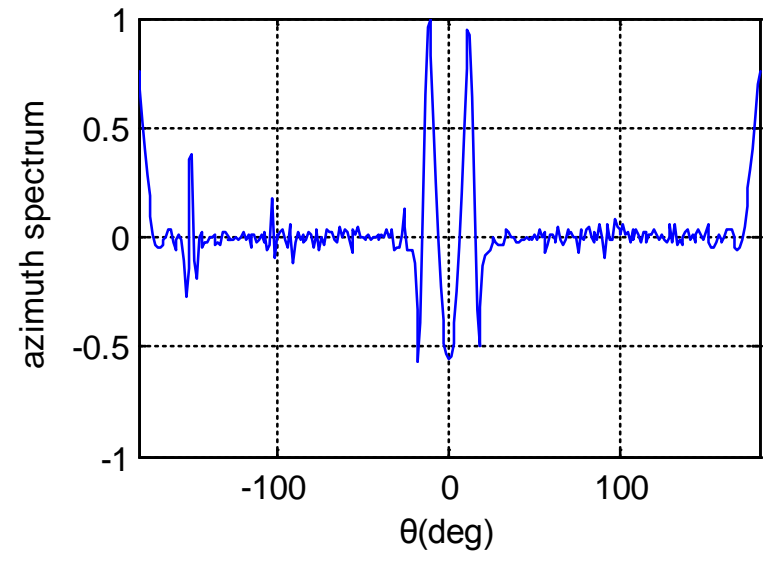

a) cardioid beamformer

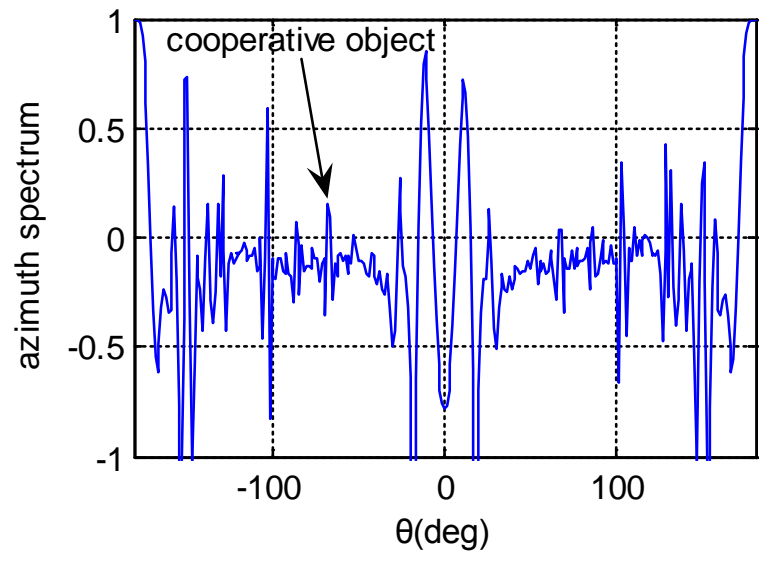

b) adaptive beamformer

Fig.6 Azimuth spectrum of cardioid beamformer and adaptive beamformer

\section{Summary}

This paper has made a simulation research on the correlation coefficient of triplet arrays under different noise conditions, and has elaborated the relationships between SNR and port-starboard discrimination performance. The results have shown that the noise correlation coefficient degrades dramatically when the flow noise of triplet arrays increases. Thus, the port-starboard discrimination performance of cardioid beamformer descends, which is based on port-starboard rejection ratio. Therefore, a method of adaptive triplet beamformer, which is based on the real time estimation of noise correlation matrix,is used to solve the problem. The adaptive beamformer uses actual estimations of the correlation coefficient to compute the port-starboard weighted values, where the correlation coefficient is obtained from the beamformed data from the received arrays. The adaptive beamformer has an advantage of the lower sidelobe and the higher ability to reject noises and jammers, except for much better port-starboard discrimination performance.Therefore, the performance of adaptive triplet beamformer is optimum to the cardioid beamformer.

\section{References}

[1] Jean Bertheas,Villeneuve Loubet. Linear hydrophonic antenna and electronic device to remove right/left ambiguity,associated with this antenna[P].United States Patent:5058082.1991-10

[2] Doisy Y. Port-starboard discriminate on performances on active towed array systems. UDT 1995 Europe,125-129P

[3] Mierlo G.W.M.van, S.P.Beerens, R.Been, Y.Doisy\&E.Trouve. Port/Starboard discrimination on hydrophone triplets in active and passive towed arrays. UDT 1997 Europe: 176-181P

[4] DU Xuan-min,ZHU Dai-zhu.Theoretical analysis and experimental research on port/starboard discrimination in towed line array.ACTA ACOUSTIC,2000,25(5):395-402p.

[5] WANG Ping-bo,ZHANG Ming-min,SHEN De-gang.Simulation Research on the port/starboard discrimination of hydrophone triplets towed line sonar by the optimum array processing algorithm in the the case of broad band.SIGNAL PROCESSING,Aug 2006,22(4):554-558p. 